\title{
On the covering dimension of a linear code
}

\author{
Thomas Britz* and Keisuke Shiromoto ${ }^{\dagger}$
}

September 16, 2018

\begin{abstract}
The critical exponent of a matroid is one of the important parameters in matroid theory and is related to the Rota and Crapo's Critical Problem. This paper introduces the covering dimension of a linear code over a finite field, which is analogous to the critical exponent of a representable matroid. An upper bound on the covering dimension is conjectured and nearly proven, improving a classical bound for the critical exponent. Finally, a construction is given of linear codes that attain equality in the covering dimension bound.

Keywords: Linear code over a finite field, Critical Problem, covering dimension, support weight distribution, characteristic polynomial.
\end{abstract}

\section{Introduction}

The Critical Problem of matroid theory, posed by Crapo and Rota [7], is to determine for $q$-representable classes of matroids $M$ the invariant $c(M ; q)$ known as the critical exponent. This problem has attracted much interest; see for instance [1, 2, 4, 15, 8, 14, 16, 18, 23, 24] and the excellent monograph-length survey [13] on this problem and variations thereof. One reason for this interest is that the critical exponent generalises the chromatic polynomial for graphs and, it was once hoped, methods for evaluating critical exponent could lead to a short and transparent proof of the famous Four Colour Theorem, which at that time was not proved to everyone's satisfaction.

These hopes have so far been frustrated since the critical exponent has turned out to be very difficult to determine, and the Critical Problem has turned out to be significantly more challenging - and of fundamental interest - than the Four Colour Problem. Until now, there are only very few general results to describe the critical exponent, one of which is the following bound by Kung [13, (4.10)]:

*School of Mathematics and Statistics, UNSW Australia, Sydney, NSW 2052, Australia (email: britz@unsw.edu.au)

${ }^{\dagger}$ Department of Mathematics and Engineering, Kumamoto University, Kurokami, Kumamoto 862-8555, Japan (email: keisuke@kumamoto-u.ac.jp) 
Theorem 1 If $M$ is a rank $k$ simple matroid that is representable over $\mathbb{F}_{q}$, then

$$
c(M ; q) \leq k-g+3,
$$

where $g$ denotes the girth of $M$, that is, the minimum cardinality of circuits of $M$.

The purpose of this paper is to introduce the covering dimension for linear codes over a finite field $\mathbb{F}_{q}$, analogous to the critical exponent for representable matroids. In Section 3 , we conjecture an upper bound on the covering dimension (cf. Conjecture 11) that improves Kung's bound above, and we nearly prove this conjecture by showing that the upper bound is true for the great majority of linear codes (cf. Corollary 21). Furthermore, in Section 4 , we provide constructions of linear codes over finite fields that attain our improved bound, thus proving that it is tight.

\section{Preliminaries}

Set $E:=\{1, \ldots, n\}$. Let $\mathbb{F}_{q}^{n}:=\mathbb{F}_{q}^{E}$ be the vector space of ordered $n$-tuples of elements from $\mathbb{F}_{q}$ indexed by $E$. The support and weight of each vector $\boldsymbol{x}=\left(x_{1}, \ldots, x_{n}\right) \in \mathbb{F}_{q}^{n}$ is given by

$$
\begin{aligned}
\operatorname{supp}(\boldsymbol{x}) & :=\left\{i: x_{i} \neq 0\right\} \\
\operatorname{wt}(\boldsymbol{x}) & :=|\operatorname{supp}(\boldsymbol{x})| .
\end{aligned}
$$

Similarly, the support and weight of each subset $B \subseteq \mathbb{F}_{q}^{n}$ are defined as follows:

$$
\begin{aligned}
\operatorname{Supp}(B) & :=\bigcup_{x \in B} \operatorname{supp}(\boldsymbol{x}) \\
\operatorname{wt}(B) & :=|\operatorname{Supp}(B)|
\end{aligned}
$$

For vectors $\boldsymbol{x}=\left(x_{1}, \ldots, x_{n}\right), \boldsymbol{y}=\left(y_{1}, \ldots, y_{n}\right) \in \mathbb{F}_{q}^{n}$, define the inner product

$$
\boldsymbol{x} \cdot \boldsymbol{y}:=\sum_{i=1}^{n} x_{i} y_{i}
$$

Let $C$ be an $[n, k]$ code $C$ over $\mathbb{F}_{q}$; that is, a $k$-dimensional subspace of the vector space $\mathbb{F}_{q}^{n}$. The dual code $C^{\perp}$ of $C$ is defined by

$$
C^{\perp}:=\left\{\boldsymbol{y} \in \mathbb{F}_{q}^{n}: \boldsymbol{x} \cdot \boldsymbol{y}=0 \text { for all } \boldsymbol{x} \in C\right\}
$$

The minimum Hamming weight of $C$ is defined by

$$
d:=d(C)=\min \{\operatorname{wt}(\boldsymbol{x}):(\mathbf{0} \neq) \boldsymbol{x} \in C\} .
$$

The minimum Hamming weight of the dual code $C^{\perp}$ is often simply denoted by $d^{\perp}:=d\left(C^{\perp}\right)$. An $[n, k, d]$ code $C$ is an $[n, k]$ code with minimum distance $d(C)=d$. 
We now introduce the covering dimension of $C$, denoted by $\gamma(C)$, as follows:

$$
\gamma(C):= \begin{cases}\infty & , \text { if } \operatorname{Supp}(C) \neq E \\ \min \left\{r \in \mathbb{Z}^{+}: A_{n}^{(r)}(C) \neq 0\right\} & , \text { otherwise }\end{cases}
$$

where $A_{i}^{(r)}(C)$ is the number of $r$-dimensional subcodes $D$ of $C$ with wt $(D)=i$.

We will now briefly show the connection between the covering dimension $\gamma(C)$ and the critical exponent $c(M ; q)$ for matroids.

For each subset $X \subseteq E$, the shortened code, denoted by $C / X$, is the linear code obtained by deleting the (zero) coordinates $X$ from each codewords $\boldsymbol{x} \in C$ with $\operatorname{supp}(\boldsymbol{x}) \cap X=\emptyset$. The punctured code, denoted by $C \backslash X$, is the linear code obtained by deleting the coordinate $X$ from each codeword in $C$. The function $\rho$ given by $\rho(X):=\operatorname{dim} C \backslash(E-X)$ for $X \subseteq E$ induces a matroid $M_{C}=(E, \rho)$ (we refer the reader to [19, 22] for information on matroids). The critical exponent $c\left(M_{C} ; q\right)$ of $M_{C}$ is defined by

$$
c\left(M_{C} ; q\right)= \begin{cases}\infty & , \text { if } M_{C} \text { has a loop; } \\ \min \left\{j \in \mathbb{Z}^{+}: p\left(M_{C} ; q^{j}\right)>0\right\} & , \text { otherwise }\end{cases}
$$

where $p\left(M_{C} ; q^{j}\right)$ is the characteristic polynomial of $M_{C}$, defined by

$$
p\left(M_{C} ; \lambda\right)=\sum_{X \subseteq E}(-1)^{|X|} \lambda^{\rho(E)-\rho(X)} .
$$

The Critical Problem of matroid theory is to determine $c\left(M_{C} ; q\right)$ (cf. [2, 4, 8, 13]). One of the main tools that has been used to address the Critical Problem is the following result widely known as the Critical Theorem by Crapo and Rota [7] (see also [4, Theorem 2]).

Theorem 2 (The Critical Theorem) Let $C$ be an $[n, k]$ code over $\mathbb{F}_{q}$. For any $X \subseteq E$ and any $m \in \mathbb{Z}^{+}$, the number of ordered $m$-tuples $\left(\boldsymbol{v}_{1}, \ldots, \boldsymbol{v}_{m}\right)$ of codewords $\boldsymbol{v}_{1}, \ldots, \boldsymbol{v}_{m}$ in $C$ with $\operatorname{supp}\left(\boldsymbol{v}_{1}\right) \cup \cdots \cup \operatorname{supp}\left(\boldsymbol{v}_{m}\right)=X$ is $p\left(M_{C /(E-X)} ; q^{m}\right)$.

By applying $E=X$ to the Critical Theorem, we can equate the covering dimension $\gamma(C)$ of a linear code over $\mathbb{F}_{q}$ and the critical exponent $c\left(M_{C} ; q\right)$ of its induced matroid $M_{C}$.

Lemma $3 \gamma(C)=c\left(M_{C} ; q\right)$.

Proof. If $c\left(M_{C} ; q\right)=m$, then the Critical Theorem implies that there is at least one set $V=\left\{\boldsymbol{v}_{1}, \ldots, \boldsymbol{v}_{m}\right\}$ of codewords in $C$ with $\operatorname{Supp}(V)=E$. Hence, $\gamma(C) \leq m=c\left(M_{C} ; q\right)$. Conversely, suppose that $\gamma(C)=m^{\prime}$. By definition, at least one $m^{\prime}$-dimensional subspace $V$ generated by $m^{\prime}$ codewords in $C$ satisfies $\operatorname{Supp}(V)=E$. Hence, $c\left(M_{C} ; q\right) \leq m^{\prime}=\gamma(C)$.

Lemma 3 implies that the covering dimension $\gamma(C)$ of a linear code is fundamentally of a set-theoretic and combinatorial nature, rather than being strictly algebraic. Conversely, the lemma lends the important observation that the critical exponent of a representable matroid $M$ does not depend on any particular linear code $C$ for which $M=M_{C}$. As a corollary to Lemma 3, and using the fact that the circuits of $M_{C}$ are also the minimal nonempty codeword supports of the dual code $C^{\perp}$ (cf. [19]), we may re-cast Theorem 1 as follows: 
Theorem 4 If $C$ is an $[n, k]$ code over $\mathbb{F}_{q}$ with $d^{\perp}:=d\left(C^{\perp}\right) \geq 3$, then

$$
\gamma(C) \leq k-d^{\perp}+3
$$

Let $A$ be an $[n, r]$ subcode of $C$ with $\operatorname{Supp}(A)=E$ having generator matrix $M G$ where $M$ is an $r \times k$ matrix over $\mathbb{F}_{q}$. Let $P$ be the $[k, r]$ code over $\mathbb{F}_{q}$ having generator matrix $M$. Then the dual code $P^{\perp}$ does not contain any of the $n$ column vectors of $G$.

Conversely, let $U$ be a $(k-r)$-dimensional subspace of $\mathbb{F}_{q}^{k}$ which does not contain any of the $n$ column vectors of $G$. Let $M$ be a generator matrix of the dual code $U^{\perp}$. Then the code $S$ having generator matrix $M G$ is an $[n, r]$ subcode of $C$ with $\operatorname{Supp}(S)=E$. Therefore we have the following correspondence (cf. [12, Lemma 2]):

Lemma 5 There exists an $[n, r]$ subcode $A \leq C$ with $\operatorname{Supp}(A)=E$ if and only if there is a $(k-r)$-dimensional subspace $U$ of $\mathbb{F}_{q}^{k}$ which contains none of the $n$ column vectors of $G$.

We summarize the above results as follows:

Proposition 6 Let $C$ be an $[n, k]$ code over $\mathbb{F}_{q}$ with generator matrix $G$.

The following are equivalent:

(1) $c\left(M_{C} ; q\right)=m$;

(2) $\gamma(C)=m$;

(3) $m$ is the smallest integer for which a $(k-m)$-dimensional subspace $U$ of $\mathbb{F}_{q}^{k}$ exists that contains no column vector of $G$.

\section{A modified bound}

\subsection{Codes attaining Kung's bound}

Let $G$ be a $k \times n$ matrix over $\mathbb{F}_{q}$ which contains as columns exactly one multiple of each nonzero vector in $\mathbb{F}_{q}^{k}$. Then the $\left[n=\left(q^{k}-1\right) /(q-1), k\right]$ code $\mathcal{H}^{\perp}$ with generator matrix $G$ is a dual Hamming code and $\left(\mathcal{H}^{\perp}\right)^{\perp}$ is a $[n, n-k, 3]$ Hamming code.

It is also known that, for each $r=1, \ldots, k$,

$$
A_{i}^{(r)}\left(\mathcal{H}^{\perp}\right)= \begin{cases}{\left[\begin{array}{l}
k \\
r
\end{array}\right]_{q}} & , \text { if } i=\left(q^{k}-q^{k-r}\right) /(q-1) ; \\
0 & , \text { otherwise }\end{cases}
$$

where $\left[\begin{array}{l}k \\ r\end{array}\right]_{q}$ denotes the Gaussian binomial coefficient (cf. [12]). We see that $i=n$ if and only if $r=k$. Therefore, $\gamma\left(\mathcal{H}^{\perp}\right)=k(=k-3+3)$, and $\mathcal{H}^{\perp}$ attains the bound in Theorem 4 .

A maximum distance separable (MDS) code over $\mathbb{F}_{q}$ is an $[n, k]$ code over $\mathbb{F}_{q}$ whose minimum Hamming weight is $n-k+1$. Since the dual code of an MDS $[n, k]$ code over $\mathbb{F}_{q}$ is also an MDS code, it follows that an $[n, k]$ code $C$ is an MDS code if and only if $d^{\perp}=k+1$. 
According to [17, Theorem 6, p. 321], the number $A_{w}$ of codewords of weight $w$ in an MDS $[n, k]$ code over $\mathbb{F}_{q}$ is given by

$$
A_{w}=\left(\begin{array}{c}
n \\
w
\end{array}\right)(q-1) \sum_{j=0}^{w-d}(-1)^{j}\left(\begin{array}{c}
w-1 \\
j
\end{array}\right) q^{w-d-j},
$$

for $d \leq w \leq n$, where $d=n-k+1$.

From Equation (2), we have that

$$
\begin{aligned}
\frac{A_{n}}{q-1} & =\sum_{j=0}^{k-1}(-1)^{j}\left(\begin{array}{c}
n-1 \\
j
\end{array}\right) q^{k-1-j} \\
& =\left(\begin{array}{c}
n-1 \\
0
\end{array}\right) q^{k-1}+\sum_{j=1}^{k-1}(-1)^{j}\left[\left(\begin{array}{c}
n-2 \\
j-1
\end{array}\right)+\left(\begin{array}{c}
n-2 \\
j
\end{array}\right)\right] q^{k-1-j} \\
& =\sum_{j=1}^{k-1}(-1)^{j}\left(\begin{array}{c}
n-2 \\
j-1
\end{array}\right) q^{k-1-j}+\sum_{j=0}^{k-1}(-1)^{j}\left(\begin{array}{c}
n-2 \\
j
\end{array}\right) q^{k-1-j} \\
& =-\sum_{j=0}^{k-2}(-1)^{j}\left(\begin{array}{c}
n-2 \\
j
\end{array}\right) q^{k-2-j}+q \sum_{j=0}^{k-2}(-1)^{j}\left(\begin{array}{c}
n-2 \\
j
\end{array}\right) q^{k-2-j}+(-1)^{k-1}\left(\begin{array}{c}
n-2 \\
k-1
\end{array}\right) \\
& =-\frac{A_{n-1}}{n(q-1)}+\frac{q A_{n-1}}{n(q-1)}+(-1)^{k-1}\left(\begin{array}{l}
n-2 \\
k-1
\end{array}\right) \\
& =\frac{A_{n-1}+(-1)^{k-1}\left(\begin{array}{l}
n-2 \\
k-1
\end{array}\right) .}{n}
\end{aligned}
$$

Therefore, if $A_{n}=0$, then $A_{n-1}=(-1)^{k} n\left(\begin{array}{l}n-2 \\ k-1\end{array}\right) \neq 0$ and $k$ is even, and so $\gamma(C)=2(=$ $k-(k+1)+3)$.

We summarize the above results as follows:

Proposition 7 If an $[n, k]$ code $C$ over $\mathbb{F}_{q}$ is a dual Hamming code or an MDS code having no codewords of weight $n$, then $C$ attains the bound in Theorem 4.

Remark 8 In [9], it is shown that if $C$ is an MDS code over $\mathbb{F}_{q}$ of length $n \leq q+2$ with no codewords of weight $n$, then $C$ is a binary $[n, n-1]$ code with odd $n$ or a $[q+1,2]$ (dual Hamming) code over $\mathbb{F}_{q}$.

In the following propositions, we classify two important classes of codes that attain the bound in Theorem 4, i.e., Kung's bound for codes. These propositions will be used in the following section.

Proposition 9 Let $C$ be a linear $[n, k]$ code over $\mathbb{F}_{q}$ with $d^{\perp}=3$. Then

$$
\gamma(C)=k-d^{\perp}+3(=k-3+3=k)
$$

if and only if $C$ is isomorphic to a dual Hamming code. 
Proof. From Proposition [6, $\gamma(C)=k$ if and only if each 1-dimensional subspace of $\mathbb{F}_{q}^{k}$ contains one column vector of a generator matrix for $C$; since $d^{\perp}=3$, any two such column vectors are linearly independent, so $C$ is isomorphic to the dual Hamming code.

Proposition 10 Let $C$ be a binary linear $[n, n-1]$ code. Then

$$
\gamma(C)=(n-1)-d^{\perp}+3(=(n-1)-n+3=2)
$$

if and only if $n$ is odd.

Proof. $C$ is isomorphic to a binary code $C^{\prime}$ having generator matrix of the form

$$
G=\left(\begin{array}{cc} 
& 1 \\
I_{n-1} & \vdots \\
& 1
\end{array}\right)
$$

Therefore, $C^{\prime}$ contains $(1, \ldots, 1,1+\cdots+1)=(1, \ldots, 1, n-1)(\bmod 2)$ as a codeword, so $\mathbf{1}=(1, \ldots, 1) \notin C$ if and only if $n$ is odd.

\section{$3.2 \quad$ A modified bound}

We now turn to the main aim of the paper which is to sharpen Kung's bound for codes (Theorem 4). In light of Proposition 7 and inspired by Remark 8 , we conjecture that Kung's bound may be sharpened as follows.

Conjecture 11 If $C$ is an $[n, k]$ code over $\mathbb{F}_{q}$ with $d^{\perp}:=d\left(C^{\perp}\right) \geq 3$, then

$$
\gamma(C) \leq k-d^{\perp}+2
$$

unless $C$ is isomorphic to a dual Hamming code or $C$ is a binary $[n, n-1]$ code such that $d^{\perp}=n$ is odd, in either which case $\gamma(C)=k-d^{\perp}+3$.

This section serves to mostly verify this conjecture. We first require two auxiliary lemmas.

Lemma 12 If $\left\{\boldsymbol{u}_{1}, \ldots, \boldsymbol{u}_{t}\right\} \subseteq \mathbb{F}_{q}^{m}$ is linearly independent, then $\left\{\boldsymbol{u}_{1}+\alpha \boldsymbol{u}_{t}, \ldots, \boldsymbol{u}_{t-1}+\alpha \boldsymbol{u}_{t}\right\}$ is also linearly independent for any $\alpha \in \mathbb{F}_{q}$.

Proof. The only scalars in $\mathbb{F}_{q}$ that satisfy

$$
\begin{aligned}
& a_{1}\left(\boldsymbol{u}_{1}+\alpha \boldsymbol{u}_{t}\right)+\cdots+a_{t-1}\left(\boldsymbol{u}_{t-1}+\alpha \boldsymbol{u}_{t}\right)=\mathbf{0}, \\
& \text { or, equivalently, } \quad a_{1} \boldsymbol{u}_{1}+\cdots+a_{t-1} \boldsymbol{u}_{t-1}+\left(\alpha \sum_{j=1}^{t-1} a_{j}\right) \boldsymbol{u}_{t}=\mathbf{0}
\end{aligned}
$$

are $a_{1}=\cdots=a_{t-1}=0$. 
Lemma 13 Let $\left\{\boldsymbol{u}_{1}, \ldots, \boldsymbol{u}_{t}\right\} \subseteq \mathbb{F}_{q}^{m}$ be linearly independent and let $D, D^{\prime}$, and $D_{0}$ be subspaces generated by $\boldsymbol{u}_{1}+\alpha \boldsymbol{u}_{t}, \ldots, \boldsymbol{u}_{t-1}+\alpha \boldsymbol{u}_{t}, \boldsymbol{u}_{1}+\beta \boldsymbol{u}_{t}, \ldots, \boldsymbol{u}_{t-1}+\beta \boldsymbol{u}_{t}$, and $\boldsymbol{u}_{1}, \ldots, \boldsymbol{u}_{t-1}$, respectively, where $\alpha, \beta \in \mathbb{F}_{q}$. Then $D=D^{\prime}$ if and only if $\alpha=\beta$; furthermore, if $\alpha \neq \beta$, then $D \cap D^{\prime} \subseteq D_{0}$.

Proof. Suppose that $D=D^{\prime}$. Then $\boldsymbol{u}_{j}+\alpha \boldsymbol{u}_{t} \in D^{\prime}$ for all $j=1,2, \ldots, t-1$, so if

$$
\begin{aligned}
\boldsymbol{u}_{j}+\alpha \boldsymbol{u}_{t} & =a_{1}\left(\boldsymbol{u}_{1}+\beta \boldsymbol{u}_{t}\right)+\cdots+a_{t-1}\left(\boldsymbol{u}_{t-1}+\beta \boldsymbol{u}_{t}\right) \\
& =a_{1} \boldsymbol{u}_{1}+\cdots+a_{j} \boldsymbol{u}_{j}+\cdots+a_{t-1} \boldsymbol{u}_{t-1}+\left(\beta \sum_{\ell=1}^{t-1} a_{\ell}\right) \boldsymbol{u}_{t}
\end{aligned}
$$

then $a_{1}=\cdots=a_{j-1}=a_{j+1}=\cdots=a_{t-1}=0, a_{j}=1$, and $\alpha=\beta$. The converse is trivial, so $D=D^{\prime}$ if and only if $\alpha=\beta$. Now suppose that $\alpha \neq \beta$. Let $\boldsymbol{v} \in D \cap D^{\prime}$ and write

$$
\begin{aligned}
\boldsymbol{v} & =a_{1}\left(\boldsymbol{u}_{1}+\alpha \boldsymbol{u}_{t}\right)+\cdots+a_{t-1}\left(\boldsymbol{u}_{t-1}+\alpha \boldsymbol{u}_{t}\right) \\
& =b_{1}\left(\boldsymbol{u}_{1}+\beta \boldsymbol{u}_{t}\right)+\cdots+b_{t-1}\left(\boldsymbol{u}_{t-1}+\beta \boldsymbol{u}_{t}\right)
\end{aligned}
$$

for some $a_{1}, \ldots, a_{t-1}, b_{1}, \ldots, b_{t-1} \in \mathbb{F}_{q}$. Then $a_{i}=b_{i}$ for all $i=1, \ldots, t-1$, and so

$$
\left(a_{1}+\cdots+a_{t-1}\right)(\alpha-\beta)=0 .
$$

We see that $a_{1}+\cdots+a_{t-1}=0$, so

$$
\begin{aligned}
\boldsymbol{v} & =a_{1}\left(\boldsymbol{u}_{1}+\alpha \boldsymbol{u}_{t}\right)+\cdots+a_{t-1}\left(\boldsymbol{u}_{t-1}+\alpha \boldsymbol{u}_{t}\right) \\
& =a_{1} \boldsymbol{u}_{1}+\cdots+a_{t-1} \boldsymbol{u}_{t-1},
\end{aligned}
$$

and we conclude that $\boldsymbol{v} \in D_{0}$.

Theorem 14 Let $C$ be a binary $[n, k]$ code with $3<d^{\perp}<k+1$. Then

$$
\gamma(C) \leq k-d^{\perp}+2 .
$$

Proof. Assume that the theorem is false for $C$. Set $t=d^{\perp}-1(<k)$. Let $\boldsymbol{g}_{1}, \ldots, \boldsymbol{g}_{t}, \boldsymbol{g}_{t+1}$ be $t+1$ linearly independent column vectors of a generator matrix $G$ of $C$. By Proposition 6 , Lemma 12, and the initial assumption, the $(t-1)$-dimensional subspace $D$ generated by $\boldsymbol{g}_{1}+\boldsymbol{g}_{t}, \ldots, \boldsymbol{g}_{t-1}+\boldsymbol{g}_{t}$ contains a column vector of $G$, say

$$
\begin{aligned}
\boldsymbol{u} & =a_{1}\left(\boldsymbol{g}_{1}+\boldsymbol{g}_{t}\right)+\cdots+a_{t-1}\left(\boldsymbol{g}_{t-1}+\boldsymbol{g}_{t}\right) \\
& =a_{1} \boldsymbol{g}_{1}+\cdots+a_{t-1} \boldsymbol{g}_{t-1}+\left(\sum_{\ell=1}^{t-1} a_{\ell}\right) \boldsymbol{g}_{t}
\end{aligned}
$$

If $a_{i}=0$ for some $i$, then $\left\{\boldsymbol{u}, \boldsymbol{g}_{1}, \ldots, \boldsymbol{g}_{i-1}, \boldsymbol{g}_{i+1}, \ldots, \boldsymbol{g}_{t}\right\}$ is linearly dependent, a contradiction. It therefore follows that $a_{i} \neq 0$ for all $i$, and so

$$
\boldsymbol{u}=\boldsymbol{g}_{1}+\cdots+\boldsymbol{g}_{t-1}+(t-1) \boldsymbol{g}_{t}(\bmod 2) .
$$


If $t$ is odd, then $\boldsymbol{u}=\boldsymbol{g}_{1}+\cdots+\boldsymbol{g}_{t-1}$ and so $\left\{\boldsymbol{u}, \boldsymbol{g}_{1}, \ldots, \boldsymbol{g}_{t-1}\right\}$ is linearly dependent, a contradiction. Therefore, $t$ is even. Similarly, the $(t-1)$-dimensional subspace $D^{\prime}$ generated by $\boldsymbol{g}_{2}+\boldsymbol{g}_{t+1}, \ldots, \boldsymbol{g}_{t}+\boldsymbol{g}_{t+1}$ contains a column vector $\boldsymbol{v}=\boldsymbol{g}_{2}+\cdots+\boldsymbol{g}_{t+1}$ of $G$. Since $\left\{\boldsymbol{g}_{1}, \ldots, \boldsymbol{g}_{t+1}\right\}$ is linearly independent, we have that $\boldsymbol{u} \neq \boldsymbol{g}_{t+1}$ and $\boldsymbol{v} \neq \boldsymbol{g}_{1}$. It follows that $\boldsymbol{u}+\boldsymbol{v}=\boldsymbol{g}_{1}+\boldsymbol{g}_{t+1}$ and so $\left\{\boldsymbol{u}, \boldsymbol{v}, \boldsymbol{g}_{1}, \boldsymbol{g}_{t+1}\right\}$ is linearly dependent. Hence, $d^{\perp} \leq 4$ and since $d^{\perp} \geq 4$, we see that $d^{\perp}=4$. However, $t$ is even, so $d^{\perp}=t+1$ is odd, a contradiction. We conclude that the theorem must be true for $C$.

Note that if $C$ is a binary $[n, k]$ code with $d^{\perp}=k+1$, then $C$ is a binary MDS code and so $k=n-1$. Therefore, by Propositions 9 and 10 and Theorem 14, we obtain the following corollary.

Corollary 15 Conjecture 11 is true for all binary linear codes.

Let use now consider linear codes over odd fields.

Lemma 16 (Theorem 11 on page 326 in [17])

If $C$ is a nontrivial $[n, k \geq 3, n-k+1] M D S$ code over $\mathbb{F}_{q}$ with $q$ odd, then $n \leq q+k-2$.

Theorem 17 Let $C$ be an $[n, k]$ code over $\mathbb{F}_{q}$ with $d^{\perp}>3$ and $q$ odd. Then

$$
\gamma(C) \leq k-d^{\perp}+2 \text {. }
$$

Proof. Assume that the theorem is false for $C$; that is, that $\gamma(C)>k-d^{\perp}+2=k-(t-1)$ where $t=d^{\perp}-1(\geq 3)$. Assume further, without loss of generality, that $G=\left[I_{k} A\right]$ is a generator matrix for $C$. By Proposition 6, we see that our assumptions imply that each $(t-1)$-dimensional subspace of $\mathbb{F}_{q}^{k}$ contains at least one of the $n$ column vectors of $G$. Let $\boldsymbol{g}_{1}, \ldots, \boldsymbol{g}_{t}$ be $t$ column vectors of $G$ and note that they are linearly independent since $d^{\perp}=t+1$. For convenience, write $\mathbb{F}_{q}=\left\{\alpha_{0}=0, \alpha_{1}, \ldots, \alpha_{q-1}\right\}$. For each $i=0,1, \ldots, q-1$, let $D_{i}$ denote the subspace generated by $\boldsymbol{g}_{1}+\alpha_{i} \boldsymbol{g}_{t}, \ldots, \boldsymbol{g}_{t-1}+\alpha_{i} \boldsymbol{g}_{t}$. From Lemmas 12 and 13 , $D_{0}, \ldots, D_{q-1}$ are mutually distinct $(t-1)$-dimensional subspaces of $\mathbb{F}_{q}^{k}$. By assumption, each subspace $D_{i}$ contains a column vector of $G$

$$
\begin{aligned}
\boldsymbol{u}_{i} & =a_{1}\left(\boldsymbol{g}_{1}+\alpha_{i} \boldsymbol{g}_{t}\right)+\cdots+a_{t-1}\left(\boldsymbol{g}_{t-1}+\alpha_{i} \boldsymbol{g}_{t}\right) \\
& =a_{1} \boldsymbol{g}_{1}+\cdots+a_{t-1} \boldsymbol{g}_{t-1}+\left(\alpha_{i} \sum_{\ell=1}^{t-1} a_{\ell}\right) \boldsymbol{g}_{t} .
\end{aligned}
$$

Now consider the matrix

$$
G^{\prime}=\left[\boldsymbol{g}_{1}, \ldots, \boldsymbol{g}_{t}, \boldsymbol{u}_{1}, \ldots, \boldsymbol{u}_{q-1}\right] .
$$

Each of the columns in $G^{\prime}$ are also columns of $G$ and we claim that these columns are distinct. Clearly, the $t$ columns $\boldsymbol{g}_{i}$ are distinct since they are linearly independent, so assume that $\boldsymbol{u}_{i}=\boldsymbol{g}_{j}$ for some $i, j$. Then

$$
\boldsymbol{g}_{j}=\boldsymbol{u}_{i}=a_{1} \boldsymbol{g}_{1}+\cdots+a_{t-1} \boldsymbol{g}_{t-1}+\left(\alpha_{i} \sum_{\ell=1}^{t-1} a_{\ell}\right) \boldsymbol{g}_{t}
$$


If $j<t$, then $a_{j}=1$ and $a_{\ell}=0$ for all $\ell \neq j$ and $\sum_{\ell=1}^{t-1} a_{\ell}=0$, a contradiction. Otherwise $j=t$, and $a_{1}=\cdots a_{t-1}=0$ and $\alpha_{i} \sum_{\ell=1}^{t-1} a_{\ell}=1$, also a contradiction. Hence, no column $\boldsymbol{u}_{i}$ is equal to any column $\boldsymbol{g}_{j}$. Finally, assume that $\boldsymbol{u}_{i}=\boldsymbol{u}_{j}$ for some distinct $i, j$; then by Lemma 13, $\boldsymbol{u}_{i} \in D_{i} \cap D_{j} \subseteq D_{0}$. Therefore, $\boldsymbol{u}_{i}$ is linearly dependent on $\boldsymbol{g}_{1}, \ldots, \boldsymbol{g}_{t-1}$, a contradiction since any $t$ columns of $G$ are linearly independent. We conclude that the columns of $G^{\prime}$ are distinct.

Since these are also columns of $G$, any $t$ of these columns are linearly independent and, for instance, the first $t+1$ column vectors are linearly dependent by construction. Since all columns of $G^{\prime}$ are contained in the span of the $t$ vectors $\boldsymbol{g}_{i}$, the dimension is exactly $t$. We see that the code $C^{\prime}$ is an $[t+q-1, t]$ MDS code.

However, $t+q-1>q+t-2$, so Lemma 16 implies that $C^{\prime}$ is not an MDS code, a contradiction.

Example 18 Let $C$ be the $[11,5]$ code over $\mathbb{F}_{3}$ having generator matrix

$$
G=\left(\begin{array}{lllllllllll}
1 & 0 & 0 & 0 & 0 & 1 & 2 & 2 & 2 & 1 & 0 \\
0 & 1 & 0 & 0 & 0 & 0 & 1 & 2 & 2 & 2 & 1 \\
0 & 0 & 1 & 0 & 0 & 2 & 1 & 2 & 0 & 1 & 2 \\
0 & 0 & 0 & 1 & 0 & 1 & 1 & 0 & 1 & 1 & 1 \\
0 & 0 & 0 & 0 & 1 & 2 & 2 & 2 & 1 & 0 & 1
\end{array}\right)
$$

Then the dual code $C^{\perp}$ is an $[11,6,5]$ quadratic residue code. By Magma calculations (cf. [3]), we have that

$$
A_{n}^{(1)}=0, \quad A_{n}^{(2)}=330, \quad A_{n}^{(3)}=825, \quad A_{n}^{(4)}=110, \quad A_{n}^{(5)}=1,
$$

where $n=11$. Therefore, $\gamma(C)=2=5-5+2=k-d^{\perp}+2$, so we see that $C$ attains equality in the bound of Theorem 17 .

Now let $C$ be an $[n, k]$ code over $\mathbb{F}_{q}$ with $d^{\perp}:=d\left(C^{\perp}\right)$. It is possible to determine many of the higher weights of $C$ as follows (cf. [10, 20]).

Lemma 19 If $k+1-d\left(C^{\perp}\right)<r \leq k$, then

$$
A_{n}^{(r)}(C)=\sum_{j=0}^{k-r}(-1)^{j}\left[\begin{array}{c}
k-j \\
k-r-j
\end{array}\right]_{q}\left(\begin{array}{l}
n \\
j
\end{array}\right) .
$$

Using the above lemma, we can verify Conjecture 11 for the cases in which $d^{\perp}=4$.

Lemma 20 Let $C$ be an $[n, k]$ code over $\mathbb{F}_{q}$ with $d^{\perp}=4$. Then

$$
\gamma(C) \leq k-d^{\perp}+2(=k-4+2=k-2) .
$$


Proof. The Singleton Bound implies that $4=d^{\perp} \leq k+1$ and so $k \geq 3$. By Lemma 19,

$$
\begin{aligned}
A_{n}^{(k-2)}(C) & =\sum_{j=0}^{2}(-1)^{j}\left[\begin{array}{l}
k-j \\
2-j
\end{array}\right]_{q}\left(\begin{array}{l}
n \\
j
\end{array}\right) \\
& =\left[\begin{array}{l}
k \\
2
\end{array}\right]_{q}-\left[\begin{array}{c}
k-1 \\
1
\end{array}\right]_{q} n+\left[\begin{array}{c}
k-2 \\
0
\end{array}\right]_{q} \frac{n(n-1)}{2} \\
& =\frac{1}{2}\left(n-\left(\left[\begin{array}{c}
k-1 \\
1
\end{array}\right]_{q}+\frac{1}{2}\right)\right)^{2}-\frac{1}{2}\left(\left[\begin{array}{c}
k-1 \\
1
\end{array}\right]_{q}+\frac{1}{2}\right)^{2}+q^{2}\left[\begin{array}{c}
k-1 \\
2
\end{array}\right]_{q}+\left[\begin{array}{c}
k-1 \\
1
\end{array}\right]_{q} \\
& =\frac{1}{2}\left(n-\left(\left[\begin{array}{c}
k-1 \\
1
\end{array}\right]_{q}+\frac{1}{2}\right)\right)^{2}+\frac{4\left(q^{k-1}-q\right)\left(q^{k-1}-1\right)-\left(q^{2}-1\right)}{8\left(q^{2}-1\right)} .
\end{aligned}
$$

We see that $A_{n}^{(k-2)}(C)>0$; hence, $\gamma(C) \leq k-2=k-d^{\perp}+2$.

By Proposition 9, Corollary 15, Theorem 17, and Lemma 20, we have proven that most cases of Conjecture 11 are true. The following corollary states this more explicitly.

Corollary 21 Conjecture 11 is true except, possibly, for linear codes over $\mathbb{F}_{q}$ where $q=2^{m}$ for some $m \geq 2$ and $d^{\perp}>4$.

Furthermore, note that Conjecture 11 is true when the size $q$ of the field $\mathbb{F}_{q}$ is sufficiently large, namely when $q \geq n$, since in that case, there is a codeword whose support is $E$, and so $\gamma(C)=1$.

To conclude this section, we will now show that Conjecture 11 is true under conditional circumstances. The first of these is the event that the following famous conjecture is true (cf. [11, p. 265]).

The MDS Conjecture Suppose that there is a nontrivial $[n, k]$ MDS code over $\mathbb{F}_{q}$. Then $n \leq q+1$, except when $q$ is even and $k=3$ or $k=q-1$ in which case $n \leq q+2$.

Theorem 22 If the MDS Conjecture is true and $d^{\perp} \neq q$, then Conjecture 11 is true.

Proof. By Corollary 21, it suffices to consider a linear code $C$ over $\mathbb{F}_{q}$ where $q=2^{m}$ for some $m \geq 2$ and $d^{\perp}>4$. Note that $t=d^{\perp}-1>3$ and that $t \neq q-1$. Assume contradictorily that $\gamma(C)>k-d^{\perp}+2=k-(t-1)$; then by the proof of Theorem 17, we may construct a $[t+q-1, t]$ MDS code $C^{\prime}$. However, the MDS Conjecture asserts that this is not possible, a contradiction.

A second conditional circumstance that implies the validity of Conjecture 11 is described in the following theorem. 
Theorem 23 Suppose that there is an $\left[n, k_{0}\right]$ code $C_{0}$ over $\mathbb{F}_{q}$ with $d\left(C_{0}^{\perp}\right)=\delta \geq 3$. If $C$ is an $[n, k]$ code over $\mathbb{F}_{q}$ with $d^{\perp}:=d\left(C^{\perp}\right)=\delta$ and $k>k_{0}$, then

$$
\gamma(C) \leq k-d^{\perp}+2
$$

Proof. Let $\boldsymbol{g}$ be a codeword of $C_{0}^{\perp}$ with wt $(\boldsymbol{g})=\delta$ and extend this codeword to form a basis $\left\{\boldsymbol{g}=\boldsymbol{g}_{1}, \ldots, \boldsymbol{g}_{n-k}\right\}$ of $C_{0}^{\perp}$. For each $i=1, \ldots, n-k-1$, let $C_{i}$ be the code generated by $\boldsymbol{g}_{1}, \ldots, \boldsymbol{g}_{n-k-i}$. Then $C_{i}$ is an $[n, n-k-i, \delta]$ code and so $C_{i}^{\perp}$ is an $[n, k+i]$ linear code with $d\left(\left(C_{i}^{\perp}\right)^{\perp}\right)=\delta$. Hence, there is an $[n, k]$ code $C\left(=C_{i}^{\perp}\right)$ over $\mathbb{F}_{q}$ with $d\left(C^{\perp}\right)=\delta$ for each $k=k_{0}, \ldots, n$.

Then let $C$ and $C^{\prime}$ be an $[n, k]$ code and an $[n, k-1]$ code, respectively, over $\mathbb{F}_{q}$ with $k_{0}<k \leq n$ and $d\left(C^{\perp}\right)=d\left(\left(C^{\prime}\right)^{\perp}\right)=\delta$. By Lemma 19,

$$
\begin{aligned}
A_{n}^{(k-\delta+2)}(C) & =\sum_{j=0}^{\delta-2}(-1)^{j}\left[\begin{array}{c}
k-j \\
\delta-2-j
\end{array}\right]_{q}\left(\begin{array}{l}
n \\
j
\end{array}\right) \\
& =\sum_{j=0}^{\delta-2}(-1)^{j}\left(\left[\begin{array}{l}
k-1-j \\
\delta-2-j
\end{array}\right]_{q}+q^{k-\delta+2}\left[\begin{array}{c}
k-1-j \\
\delta-3-j
\end{array}\right]_{q}\right)\left(\begin{array}{l}
n \\
j
\end{array}\right) \\
& =\sum_{j=0}^{\delta-2}(-1)^{j}\left[\begin{array}{l}
k-1-j \\
\delta-2-j
\end{array}\right]_{q}\left(\begin{array}{l}
n \\
j
\end{array}\right)+q^{k-\delta+2} \sum_{j=0}^{\delta-3}(-1)^{j}\left[\begin{array}{l}
k-1-j \\
\delta-3-j
\end{array}\right]_{q}\left(\begin{array}{l}
n \\
j
\end{array}\right) \\
& =A_{n}^{(k-\delta+1)}\left(C^{\prime}\right)+q^{k-\delta+2} A_{n}^{(k-1-\delta+3)}\left(C^{\prime}\right) .
\end{aligned}
$$

By Theorem 4, $A_{n}^{(k-\delta+2)}(C) \geq q^{k-\delta+2} A_{n}^{(k-1-\delta+3)}\left(C^{\prime}\right)>0$. Hence, $\gamma(C) \leq k-d^{\perp}+2$.

\section{A construction of minimal blocks}

In this section, we present an infinite class of linear codes that each attains the bound in Conjecture 11.

As defined in [15, 21], a set $M$ of points of the projective geometry $P G(k-1, q)$ is an $r$-block over $\mathbb{F}_{q}$ for some integer $r$ with $1 \leq r \leq k-1$ if every $(k-r)$-dimensional subspace in $P G(k-1, q)$ contains at least one point in $M$. If $X$ is a flat in $M$, a tangent of $X$ is a $(k-r)$-dimensional subspace $U$ in $P G(k-1, q)$ such that

$$
M \cap U=X .
$$

An $r$-block $M$ is minimal if every point in $M$ has a tangent, and to be tangential if every proper nonempty flat in $M$ of rank not exceeding $k-r$ has a tangent.

Alternatively, a matroid $M$ is a tangential $r$-block over $\mathbb{F}_{q}$ if the following conditions hold:

(i) $M$ is simple and representable over $\mathbb{F}_{q}$.

(ii) $p\left(M ; q^{r}\right)=0$.

(iii) $p\left(M / F ; q^{r}\right)>0$ whenever $F$ is a proper nonempty flat of $M$. 
A construction of minimal blocks from binary vectors is given in [15].

In the following, let $k$ and $m$ be positive integers with $m \leq k$. Set $K:=\{1, \ldots, k\}$ and let $T \in\left(\begin{array}{l}K \\ m\end{array}\right)$. Also, suppose that $\mathcal{V}$ is a family of $m-1$ distinct points $\boldsymbol{v}_{1}, \ldots, \boldsymbol{v}_{m-1} \in P G(k-1, q)$ with $\operatorname{supp}\left(\boldsymbol{v}_{i}\right) \cap T=\emptyset$ for each $i=1, \ldots, m-1$. Define

$$
\begin{aligned}
X^{T} & :=\{\boldsymbol{x} \in P G(k-1, q): \operatorname{supp}(\boldsymbol{x}) \cap T=\emptyset\}, \\
Y_{\mathcal{V}}^{T} & :=\{\boldsymbol{x} \in P G(k-1, q):|\operatorname{supp}(\boldsymbol{x}) \cap T|=1\} \backslash \bigcup_{j \in T} \bigcup_{\boldsymbol{v}_{i} \in \mathcal{V}}\left\{\boldsymbol{v}_{i}+\lambda \boldsymbol{e}_{j}: \lambda \in \mathbb{F}_{q}-\{0\}\right\}, \\
Z^{T} & :=\left\{\boldsymbol{x} \in P G(k-1, q): \operatorname{supp}(\boldsymbol{x}) \in\left(\begin{array}{c}
T \\
2
\end{array}\right)\right\}, \\
M & :=X^{T} \cup Y_{\mathcal{V}}^{T} \cup Z^{T},
\end{aligned}
$$

where $\boldsymbol{e}_{i}$ denotes the vector in $\mathbb{F}_{q}^{k}$ with a 1 in the $i$ th coordinate and 0's elsewhere.

Theorem $24 M$ is a $(k-m)$-block over $\mathbb{F}_{q}$.

To prove this theorem, we need the following lemma.

Lemma 25 For any $j \in T$, define

$$
M_{j}:=\{\boldsymbol{x} \in M: j \in \operatorname{supp}(\boldsymbol{x})\} .
$$

Then the following hold:

(1) $\left|M_{j}\right|=q^{k-m}$.

(2) For any distinct points $\boldsymbol{x}, \boldsymbol{y} \in M_{j}$, there exist $\alpha, \beta \in \mathbb{F}_{q}$ such that $\alpha \boldsymbol{x}+\beta \boldsymbol{y} \in M$.

Proof. (1) From the definition of $X^{T}, Y_{\mathcal{V}}^{T}$, and $Z^{T}$, we have that

$$
\begin{aligned}
& \left|X^{T} \cap M_{j}\right|=0, \\
& \left|Y_{\mathcal{V}}^{T} \cap M_{j}\right|=(q-1)(|P G(k-m-1, q)|-(m-1))+1=q^{k-m}-(q-1)(m-1), \\
& \left|Z^{T} \cap M_{j}\right|=(q-1)(m-1) .
\end{aligned}
$$

The equation follows.

(2) Write ${ }^{t} \boldsymbol{x}=\left(x_{1}, \ldots, x_{k}\right),{ }^{t} \boldsymbol{y}=\left(y_{1}, \ldots, y_{k}\right)$, and $\boldsymbol{z}=x_{j}^{-1} \boldsymbol{x}-y_{j}^{-1} \boldsymbol{y}$, and note that $\boldsymbol{x}, \boldsymbol{y} \notin X^{T}$ and that $j \notin \operatorname{supp}(\boldsymbol{z})$. If $\boldsymbol{x}, \boldsymbol{y} \in Y_{\mathcal{A}}^{T}$, then $\operatorname{supp}(\boldsymbol{z}) \cap T=\emptyset$ and so $\lambda \boldsymbol{z} \in X^{T} \subseteq M$ for some $\lambda \in \mathbb{F}_{q}-\{0\}$. Next, suppose that $\boldsymbol{x} \in Y_{\mathcal{V}}^{T}$ and $\boldsymbol{y}=y_{j} \boldsymbol{e}_{j}+y_{\ell} \boldsymbol{e}_{\ell} \in Z^{T}$ and note that $\boldsymbol{x}-\mu^{\prime} \boldsymbol{e}_{j} \neq \mu \boldsymbol{v}_{i}$ for each $\boldsymbol{v}_{i} \in \mathcal{V}$ and any $\mu, \mu^{\prime} \in \mathbb{F}_{q}-\{0\}$. Then $\operatorname{supp}(\boldsymbol{z}) \cap T=\{\ell\}$ and $\boldsymbol{z}+y_{j}^{-1} y_{\ell} \boldsymbol{e}_{\ell}=x_{j}^{-1}\left(\boldsymbol{x}-x_{j} \boldsymbol{e}_{j}\right) \neq \mu \boldsymbol{v}_{i}$ for any $\boldsymbol{v}_{i} \in \mathcal{V}$ and any $\mu \in \mathbb{F}_{q}-\{0\}$, Hence, $\lambda \boldsymbol{z} \in Y_{\mathcal{V}}^{T} \subseteq M$ for some $\lambda \in \mathbb{F}_{q}-\{0\}$. By symmetry, the same is true if $\boldsymbol{x} \in Z^{T}$ and $\boldsymbol{y} \in Y_{\mathcal{V}}^{T}$. Finally, suppose that $\boldsymbol{x}, \boldsymbol{y} \in Z^{T}$. Since $\boldsymbol{x}$ and $\boldsymbol{y}$ are distinct, the $\operatorname{support} \operatorname{supp}(\boldsymbol{z})$ consists of either one or two elements of $T$, and so $\lambda \boldsymbol{z} \in Y_{\mathcal{V}}^{T} \cup Z^{T} \subseteq M$ for some $\lambda \in \mathbb{F}_{q}-\{0\}$.

In each case, $\alpha \boldsymbol{x}+\beta \boldsymbol{y}=\lambda \boldsymbol{z} \in M$ with $\alpha=\lambda x_{j}^{-1}$ and $\beta=-\lambda y_{j}^{-1}$ for some $\lambda \in \mathbb{F}_{q}-\{0\}$. 
Proof of Theorem 24. Suppose that there exists a $(k-m) \times k$ matrix $H=\left[\boldsymbol{a}_{1}, \ldots, \boldsymbol{a}_{k}\right]$ over $\mathbb{F}_{q}$ of rank $k-m$ such that

$$
M \cap\{\boldsymbol{y} \in P G(k-1, q): H \boldsymbol{y}=\mathbf{0}\}=\emptyset .
$$

Choose any $j \in T$. For any point $\boldsymbol{x}={ }^{t}\left(x_{1}, \ldots, x_{k}\right) \in M_{j}, H \boldsymbol{x} \neq \mathbf{0}$ and so it follows that

$$
\boldsymbol{a}_{j} \neq-x_{j}^{-1} \sum_{\ell \neq j} x_{\ell} \boldsymbol{a}_{\ell} .
$$

For any distinct points $\boldsymbol{x}={ }^{t}\left(x_{1}, \ldots, x_{k}\right), \boldsymbol{y}={ }^{t}\left(y_{1}, \ldots, y_{k}\right) \in M_{j}$, Lemma 25 (2) implies that $H\left(x_{j}^{-1} \boldsymbol{x}-y_{j}^{-1} \boldsymbol{y}\right) \neq \mathbf{0}$ and so

$$
-x_{j}^{-1} \sum_{\ell \neq j} x_{\ell} \boldsymbol{a}_{\ell} \neq-y_{j}^{-1} \sum_{\ell \neq j} y_{\ell} \boldsymbol{a}_{\ell} .
$$

By Lemma 25 (1), we have that

$$
\left|\left\{-x_{j}^{-1} \sum_{\ell \neq j} x_{\ell} \boldsymbol{a}_{\ell}: \boldsymbol{x}={ }^{t}\left(x_{1}, \ldots, x_{k}\right) \in M_{j}\right\}\right|=\left|M_{j}\right|=q^{k-m} .
$$

By (3) and (44), the column vector $\boldsymbol{a}_{j}$ is not in $\mathbb{F}_{q}^{k-m}$, a contradiction.

Theorem 26 Let $M$ be the set of points in $P G(k-1, q)$ defined in Theorem 24 . If $m \leq q^{k-m-1}$, then $M$ is a minimal $(k-m)$-block over $G F(q)$.

Proof. Without loss of generality, set $T:=\{k-m+1, \ldots, k\}$. Choose any point $\boldsymbol{x}={ }^{t}\left(x_{1}, \ldots, x_{k}\right) \in M$. We first consider the case $\boldsymbol{x} \in X^{T}$. Set $\ell:=\max \left\{j \in K: x_{j} \neq 0\right\}$ and note that $\ell \leq m-k$. Consider a $(k-m) \times k$ matrix

$$
H=\left[\boldsymbol{e}_{1}, \ldots, \boldsymbol{e}_{\ell-1}, \boldsymbol{b}, \boldsymbol{e}_{\ell+1}, \ldots, \boldsymbol{e}_{k-m}, \boldsymbol{y}_{0}, \boldsymbol{y}_{1}, \ldots, \boldsymbol{y}_{m-1}\right]
$$

where

$$
\boldsymbol{b}= \begin{cases}0 & , \text { if } \operatorname{wt}(\boldsymbol{x})=1 \\ -x_{\ell}^{-1} \sum_{j=1}^{\ell-1} x_{j} \boldsymbol{e}_{j} & , \text { otherwise }\end{cases}
$$

and $\boldsymbol{y}_{0}=\boldsymbol{e}_{\ell}, \boldsymbol{y}_{1}, \ldots, \boldsymbol{y}_{m-1}$ are mutually distinct points in

$$
\left\{\boldsymbol{z}+\boldsymbol{e}_{\ell}: \boldsymbol{z} \in \mathbb{F}_{q}^{k}, \operatorname{supp}(\boldsymbol{z}) \cap(T \cup\{\ell\})=\emptyset\right\} .
$$

We note that there always exist these $m$ points whenever $m \leq q^{k-m-1}$. Let $U$ be the null space in $P G(k-1, q)$ of the matrix $H$. Then $\boldsymbol{x} \in U$ but $\boldsymbol{y} \notin U$ for any $\boldsymbol{y} \in M-\{\boldsymbol{x}\}$.

Next we consider the case $\boldsymbol{x} \in Y_{\mathcal{V}}^{T}$. We may assume without loss of generality that $\operatorname{supp}(\boldsymbol{x}) \cap T=\{k-m+1\}$ and write ${ }^{t} \boldsymbol{v}_{i}=\left(v_{1}^{(i)}, \ldots, v_{k}^{(i)}\right)$ for any point $\boldsymbol{v}_{i}$ in the family $\mathcal{V}$. Consider a $(k-m) \times k$ matrix

$$
H=\left[\boldsymbol{e}_{1}, \ldots, \boldsymbol{e}_{k-m}, \boldsymbol{c}, \boldsymbol{w}_{1}, \ldots, \boldsymbol{w}_{m-1}\right]
$$


where

$$
\begin{aligned}
\boldsymbol{c} & =-x_{k-m+1}^{-1} \sum_{j=1}^{k-m} x_{j} \boldsymbol{e}_{j}, \\
\boldsymbol{w}_{i} & =\sum_{j=1}^{k-m} v_{j}^{(i)} \boldsymbol{e}_{j}, \quad \text { for } i=1, \ldots, m-1 .
\end{aligned}
$$

Then the null space $U$ of $H$ is a tangent for $\boldsymbol{x}$.

Finally, consider the case in which $\boldsymbol{x} \in Z^{T}$. Assume without loss of generality that $\operatorname{supp}(\boldsymbol{x})=\{k-m+1, k-m+2\}$; then we can construct the null space $U$ by replacing $\boldsymbol{c}$ by $-x_{k-m+1}^{-1} x_{k-m+2} \boldsymbol{w}_{1}$ in the above matrix $H$.

By definition, $M$ is a minimal $r$-block over $\mathbb{F}_{q}$ if and only if $\gamma(C)=r+1$ for the linear code $C$ having generator matrix $G$ whose column vectors are all points in $M$ (cf. [6, p. 168]).

Corollary 27 Let $M$ be the set of points defined in Theorem 24 with $m=2$, and let $C$ be the linear code over $\mathbb{F}_{q}$ whose generator matrix is obtained from $M$. Then $C$ attains the bound in Conjecture 11 .

Proof. From the definition of $M$, we see that $d^{\perp}=3$ since there are three linearly dependent column vectors in $G$. Thus,

$$
k-2+1=k-1=\gamma(C) \leq k-3+2=k-1 .
$$

\section{References}

[1] R.M. Ankney and J.E. Bonin, Characterisations of $P G(n-1, q) \backslash P G(k-1, q)$ by numerical and polynomial invariants, Adv. Appl. Math. 28 (2002), 287-301.

[2] T. Asano, T. Nishizeki, J. Oxley, and N. Saito, A note on the critical problem for matroids, European J. Combin. 5 (1984), 93-97.

[3] W. Bosma, J.J. Cannon, C. Fieker, A. Steel (eds.), Handbook of Magma Functions, Edition 2.16 (2010), 5017 pages.

[4] T. Britz, Extensions of the critical theorem, Discrete Math. 305 (2005), 55-73.

[5] T. Britz, Code enumerators and Tutte polynomials, IEEE Trans. Inform. Theory 56 (2010), 4350-4358.

[6] T. Brylawski and J. Oxley, The Tutte polynomial and its applications, in Matroid applications, pp. 123-225, Cambridge Univ. Press, Cambridge, 1992.

[7] H.H. Crapo and G.-C. Rota, On the Foundations of Combinatorial Theory: Combinatorial Geometries (Preliminary Edition), The M.I.T. Press, Cambridge, Mass.-London, 1970. 
[8] T.A. Dowling, Codes, packings and the critical problem, in Applicazioni (Univ. Perugia, Perugia, 1970), pp. 209-224. Ist. Mat., Univ. Perugia, Perugia, 1971.

[9] M.F. Ezerman, M. Grassl and P. Solé, The weights in MDS codes, IEEE Trans. Inform. Theory 57 (2011), 392-396.

[10] T. Helleseth, T. Kløve, and J. Mykkeltveit, The weight distribution of irreducible cyclic codes with block lengths $n_{1}\left(\left(q^{l}-1\right) / N\right)$, Discrete Math. 18 (1977), 179-211.

[11] W.C. Huffman and V. Pless, Fundamentals of Error-Correcting Codes, Cambridge University Press, 2003.

[12] T. Kløve, Support weight distribution of linear codes, Discrete Math. 106/107 (1992), $311-316$.

[13] J.P.S. Kung, Critical problems, in: Matroid Theory, Seattle, WA, 1995, Contemporary Mathematics, 197, American Mathematical Society, Providence, RI, 1996, pp. 1-127.

[14] J.P.S. Kung, Critical exponents, colines, and projective geometries, Combin. Probab. Comput. 9 (2000), 355-362.

[15] J.P.S. Kung, Minimal blocks of binary even-weight vectors, Linear Algebra Appl. 416 (2006), 288-297.

[16] B. Lindström, On the chromatic number of regular matroids, J. Combin. Theory, Ser. B 24 (1978), 367-369.

[17] F.J. MacWilliams and N.J.A. Sloane, The Theory of Error-Correcting Codes, Amsterdam, The Netherlands: North-Holland, 1977.

[18] J. Oxley, Colouring, packing and the critical problem, Quart. J. Math. Oxford (2) 29 (1978), 11-22.

[19] J. Oxley, Matroid Theory, Oxford University Press, New York, 1992.

[20] H.G. Schaathun, Support Weights in Linear Codes and Projective Multisets, ph.d. thesis, University of Bergen, 2001.

[21] P.N. Walton and D.J.A. Welsh, Tangential 1-blocks over GF(3), Discrete Math. 40 (1982), 319-320.

[22] D.J.A. Welsh, Matroid Theory, Academic Press, London, 1976.

[23] G. Whittle, On the critical exponent of transversal matroids, J. Combin. Theory Ser. B 37 (1984), 94-95,

[24] G. Whittle, Dowling group geometries and the critical problem, J. Combin. Theory Ser. B 47 (1989), 80-92. 